\title{
Management Prospects for COVID-19: A Review
}

\author{
Uzma Aamir Jilani, Ishrat Rahman, Syed Aamir Jilani \\ Uzma Aamir Jilani [Jilani UA] \\ Postgraduate Biomedicine Research student \\ Management \& Science University, Malaysia \\ jilanies@yahoo.com
}

Ishrat Rahman [Rahman I]

Assistant Professor of Pharmacology

College of Dentistry

Princess Nourah bint Abdulrahman University, Riyadh, KSA

imrahman@pnu.edu.sa

Syed Aamir Jilani [Jilani SA]

Dublin Business School, Ireland

Draamir.jilani@gmail.com

Corresponding Author: Dr Ishrat Rahman imrahman@pnu.edu.sa

\begin{abstract}
Although the number of active COVID-19 cases have been fallen in some countries, the threat of the pandemic is still impending in many parts of the world and the pace of recovery is still uncertain. SARS-CoV-2 outbreak has expanded very quickly since the beginning of 2020 and has now taken approximately a million lives all around the globe. Sadly, there is yet to be an approved treatment for COVID-19 as it may take a few years to establish a definitive treatment and a vaccine against SARS-CoV-2. This study aims to review the preventive and curative measures currently under investigation for COVID-19 and the targeted pathogenic features of the SARS-CoV-2. To manage the clinical implications of COVID-19, and to alleviate SARS$\mathrm{CoV}-2$, the main focus is on supportive therapy, symptom control, antiviral drugs, and immunomodulation. Intense research activities for therapeutic interventions are in progress worldwide to evaluate the benefit of such drugs.
\end{abstract}

Keywords. COVID-19, SARS-CoV-2, Prevention, Treatment, Antiviral therapy, Immunomodulators. 


\section{Introduction}

The members of coronavirus family have caused three major outbreaks in the past twenty years, commencing with the Severe Acute Respiratory Syndrome (SARS) in 2002 caused by SARS-CoV, followed by Middle Eastern Respiratory Syndrome (MERS) in 2012 caused by MERS-CoV, and now the most contagious Coronavirus Disease 2019, named as COVID-19 caused by SARS-CoV-2. [1] The initial cases of pneumonia caused by SARS-CoV-2 were reported in Wuhan, China in December 2019 and within a short period, the virus spread around the world and became a public threat. [2] It was declared a pandemic on 11th March 2020 by the World Health Organisation. Although the genetic sequence of SARS-CoV-2 and its pathogenic characteristics were shared by the Chinese Health Authorities in the least possible time the containment of the virus remains a challenge to date.[3] Effective preventive measures are required in affected countries to break the chain of infection as no specific therapeutic agent has been approved for use in clinical practice to alleviate the virus and no vaccine is available to produce the herd immunity. [4] So far approximately thirty million people have been affected and the numbers are going to rise. [5] The main concern right now should be how to control a pandemic that has no end in sight. Considering the previous experiences of SARS-CoV and other viral diseases, some potential therapeutic agents such as hydroxychloroquine, interferons, nucleoside analogues, remdesivir, lopinavir/ritonavir, RNA synthesis inhibitors, convalescent plasm, tocilizumab, and other anti-inflammatory drugs have been administered to COVID-19 patients and their efficacy and safety were monitored.[6] Some of these drugs like Hydroxychloroquine and Lopinavir/Ritonavir have been recently disapproved as a therapy to COVID-19 by the WHO after receiving results from several clinical trials, which observed insignificant or no benefits in terms of the disease outcome. [7] The others are still to be approved or disapproved as an effective treatment to COVID-19. In this narrative review, management options including preventive and therapeutic measures to alleviate SARS-CoV-2 currently in practice and under experimental stage are recapitulated. The genetic, virulent, and transmission properties of the novel virus which laid the targets for prevention and compassionate use of repurposed drugs are also reported.

\section{Method}

A swift review was performed by using Ovid Medline and the Cochrane Database of systematic reviews from 1 January 2020 to 31 August 2020. Latest updates through the WHO COVID-19 publications and clinicaltrials.gov were also searched and reviewed. The terms used for the literature search were COVID-19, treatment options, antiretroviral, protease inhibitors, convalescent plasma, ACE receptor blockers, antimalarials, immunomodulators, and monoclonal antibodies. Some relevant citations were identified through snowballing and by utilizing the 'cited by' function available on the Google Scholar. Finally, a total of 46 studies were screened and reviewed, all of these studies and clinical trials were in the English language.

\section{SARS CoV2 Virulence and Pathogenesis}

The novel SARS-CoV-2 like SARS-Co-V and MERS-Co-V, belong to the $\beta$-coronaviruses group. The potential natural reservoir of the SARS-CoV-1 and SARS-CoV-2 is identified as a bat and both enveloped, RNA viruses share $80 \%$ genomic similarity [8]. It is assumed that a mutation in the genetic sequence of coronaviruses resulted in the SARS-CoV-2 outbreak.[3] According to research interventions, there is a significant number of amino acid substitutions both in the structural and nonstructural proteins between the SARS-CoV-2 and previous human coronaviruses. [8] The mechanism of this genetic mutation is still inconclusive and might be the cause for increased virulence of SARS$\mathrm{CoV}-2$ as compared to other coronaviruses. [9] 
SARS-CoV-2 spread in Wuhan, China in December 2019 by zoonotic transmission. However, later it spread mainly through droplet transmission.[10] Clinically active cases of COVID-19 are the main reservoir and spread the virus by sneezing and coughing. People in close contact such as family members and medical staff are the most vulnerable.[11] SARS-CoV-2 remains viable for up to 4 to several hours on different surfaces, ranging from copper to plastics respectively and therefore vector transmission is another possible route of transmission. [11]

Various studies have confirmed that ACE2 is a functional receptor for SARS-CoV-2 like SARSCoV-1 and both use the same receptor for making their way into the host cell. [12] This feature may contribute to developing a therapy to alleviate the virus by blocking the receptor.[13] After penetrating the host cell, the virus starts replicating and finally released into the system. During this process, viral antigens are exposed to antigen-presenting cells and a cascade of inflammatory response initiated. [14] A bizarre production of proinflammatory and anti-inflammatory chemical mediators is found to be the main cause of pathology in COVID-19 cases. Increased production of various interleukins (IL-1, IL-6, IL-7, IL-10), interferon-gamma, and tumour necrosis factor-alpha (TNF-alpha) through a chain of reactions, by activated macrophages and T-helper cells, results in a cytokine storm. [14] This hyperimmune response is responsible for causing severe pneumonia and acute respiratory distress syndrome (ARDS) in critical cases. [15] Further expansion of immunological response interferes with the functions of multiple vital organs and results in multiple organ failure. Immunomodulation through immunosuppressive therapy and corticosteroids therefore helps in reducing the cytochemical and inflammatory havoc caused by SARS-CoV-2.[16] Thus, improving the clinical symptoms and signs in critical cases of COVID-19, reducing the severity of disease and mortality rate.

\section{Clinical manifestations}

Based on the severity of the disease the clinical presentation of COVID-19 is divided into four types. [17] Mild illness comprised of general symptoms such as fatigue, fever, headache, myalgia, loss of taste and smell sensation, with the disease limited to upper respiratory tract only. An infected person might present with a sore throat, nasal congestion, and dry cough.[18] The moderate disease is associated with the involvement of the lower respiratory tract, marked with productive cough, breathing difficulty, chest pain, anorexia, and confusion. [19] Patients presenting with signs and symptoms of acute respiratory distress syndrome such as tachypnoea, painful and laboured breathing, fever, and cyanosis are marked as severely infected. [20] The critical disease is associated with respiratory failure, metabolic acidosis, and multiple organ dysfunction. [12] Acute cardiac events such as ischemia and arrhythmia, acute renal failure, septicaemia, and nosocomial infections, have been reported in critically ill COVID-19 patients. [21]

\section{Therapeutic Options for COVID-19}

There are still many uncertainties on the treatment options available to alleviate SARS-CoV-2. In mild and moderate cases symptomatic treatment by using conventional therapies like analgesics and antipyretics is recommended with adequate hydration. Management options for severe and critical cases consist of supportive care, including non-invasive and invasive oxygen therapy depending upon the severity of the clinical condition, and compassionate use of various therapeutic agents. [22] Repurposed drugs and vaccines are under trial to combat SARS-CoV-2, such as immunomodulators (chloroquine and hydroxychloroquine), protease inhibitors (lopinavir and ritonavir), monoclonal antibodies, peptides, Interferon-based therapies, immunosuppressors to suppress the immune response, and ACE-2 receptor blockers (camostat mesylate) are among them. [23,24] All these potential therapies are yet to establish as an effective management solution to COVID-19. 


\subsection{Role of Antibiotics and Corticosteroids.}

In hospitalized cases of COVID-19 and those on mechanical ventilation, provisional use of broadspectrum antibiotics to manage secondary bacterial respiratory tract infections, have been recommended, whereas the use of corticosteroids remains questionable. [25] The commonly used corticosteroid, Dexamethasone is widely available and its use as a life-saving drug is approved by the Food and Drug Administration (FDA) and WHO for many severe clinical conditions such as anaphylactic shock and other autoimmune and hypersensitivity conditions. Recently it has been confirmed through the RECOVERY trial (NCT04381936) conducted by Oxford University, UK, that Dexamethasone has proven effective in critically ill COVID-19 cases and reduces death rate. [26,27] The main mechanism of action is to curtail inflammatory mediators and hence reduce oedema and tissue damage in such cases. [16] In critical cases of SARS-CoV-2 pneumonia, the airways get filled with exudate due to cytokine storm, reducing gaseous transfer and oxygen saturation in the blood. In such cases, corticosteroids have found to be beneficial and reducing the severity as well as the death rate of the disease. However, these cases need intensive care surveillance, blood glucose levels, electrolytes, liver functions and blood oxygen saturation monitoring. Direct oxygen therapy, extracorporeal membrane oxygenation (ECMO), and protective mechanical ventilation are recommended in cases of hypoxemia, for supportive care which relieves respiratory distress, reduces cardiovascular complications, as well as death rate. [12]

\section{2 'Solidarity' clinical trial by $\mathrm{WHO}$.}

WHO has started an international clinical trial with the name, 'Solidarity' to coordinate the scientific data collection on many of the most recommended repurposed drugs for use to treat SARS-COV-2 infection. It is a multi-arm "mega-trial" that aims to assess and compare the effectiveness of four treatment options against COVID-19, currently active in many parts of the world and will accelerate the effective drug discovery process to combat COVID-19 crises. The drugs initially included in the Solidarity clinical trial were Remdesivir, Lopinavir/ritonavir combined, Lopinavir/ritonavir combined with interferon-beta, and Hydroxychloroquine or chloroquine. WHO is tracking disease progression in patients under these treatment options enrolled in multiple countries by the collaboration of authorised agencies. [7] Recently Hydroxychloroquine and Lopinavir/ritonavir have been withdrawn from the Solidarity clinical trial as both drugs have not shown any improvement or benefit in the COVID-19 outcome. [7] However, their use as a prophylactic agent for COVID-19 in non-hospitalized patients remains unaffected.

\subsection{Remdesivir.}

Remdesivir is a potential repurposed therapy for COVID-19. Multiple clinical trials are currently underway or in progress concerning the use of Remdesivir against SARS-CoV-2. It is an antiviral drug initially used as a possible treatment for Ebola Haemorrhagic Virus (EBV). It is a nucleotide analogue, proved to have broad-spectrum activity against the single-stranded RNA viruses. Current research trials of the drug on COVID-19 has revealed that it is effective against certain key enzymes of SARS-CoV-2, thus, stopping the replication mechanism of the virus. [28] It was observed that Remdesivir, stops the polymerase enzyme from adding more RNA subunits, halting genome replication. Various clinical trials all over the world suggest that compassionate use of remdesivir may have clinical benefit in severe cases of Covid-19. [29,30]

\subsection{Lopinavir/ Ritonavir.}

Coronaviruses have the largest genomes among all the RNA viruses. The virulence of the virus depends upon the encoding of structural proteins and mainly on the transcription and translation of the viral 
genome into a polypeptide. This polypeptide encodes specific proteins that are responsible for viral replication and gene expression. An enzyme is required for the replication of the coronavirus which is known as $\sim 306$ aa long main protease (Mpro) and is encoded by the polypeptide. [31] Once encoded it converts the polypeptide into functional proteins. It is observed that Mpros of different coronaviruses share great structural and sequential similarity and therefore researchers target Mpro by using protease inhibitors as anti-coronaviral drugs. Lopinavir is an antiretroviral drug used for HIV-1 which binds to the same target site of Mpro and thus inhibit the viral replication by blocking the protease enzyme. [32] It is used in combination with Ritonavir another antiviral, the main function of which is to increase the half-life of Lopinavir. Since the beginning of pandemic combination of lopinavir and ritonavir is advocated as a potential therapy for COVID-19 and was included in the WHO solidarity trial, but due to insignificant outcomes in terms of reduction of mortality and severity, it is now excluded from the WHO solidarity trial.

\subsection{Hydroxychloroquine (HCQ).}

HCQ, an antimalarial drug approved by the WHO as one of the safest, effective, and essential therapy for healthcare provision. It is used for many clinical conditions besides malaria, such as rheumatoid arthritis, Systemic lupus erythematosus, porphyria, etc. Since the beginning of the SARS-CoV-2 outbreak, HCQ has been on the front runners-up as a potential therapeutic remedy for COVID-19. It is speculated that HCQ possess antiviral activity through immunomodulatory effects on antigen-presenting cells and disrupts the binding activity of the viral spike proteins with the ACE2 receptors on the pulmonary host cells. [33] HCQ is part of the various clinical trials such as 'Recovery Trial' by Oxford University UK, and 'Solidarity' by the WHO. However, due to inefficacy and safety concerns, it is revoked from the Solidarity trial since June. The drug is still under experimental use for prophylaxis and treatment of COVID-19 in many countries. [34]

\subsection{Interferons (IFNs).}

IFNs are synthesized by recombinant DNA technology and various clinically approved subtypes are available.[15] Type1 IFN- IFN alpha/beta belong to cytokine family and they exhibit antiviral properties and are used as broad-spectrum antivirals. These chemical mediators have autocrine and paracrine functions and potentiate the exposition of interferon-stimulated genes (ISG). The ISGs transmit the antiviral properties to host cells to fight against the viruses.[35] IFNs inhibit viral replication and support immune response to eradicate the virus. Previous experience with SARS-CoV outbreak (2003) proved that IFN-alpha accentuated the clearance of lung pathology caused by SARS-CoV in critically ill patients. Recent clinical trials of IFN-beta in COVID-19 cases have shown improved survival when administered in the early phase of the disease and reduced hospital stay and mortality, supplementing the standard of care. [36]

\subsection{Camostat Mesylate.}

Camostat Mesylate is an orally bioavailable, synthetic protease inhibitor, with anti-inflammatory, and potential antiviral activities. It was developed and used in Japan since 1980 for the treatment of acute pancreatitis and reflux esophagitis. It inhibits the activities of a variety of proteases, including trypsin, kallikrein, thrombin, plasmin, and some complement system enzymes like $\mathrm{C} 1$ esterase. Camostat Mesylate may also suppress the production and activity of cytokines such as IL-1, IL-6, TNF-alpha and transforming growth factor-beta (TGF-beta). This reduces inflammation and alleviates the cytokine storm. Besides, this compound may inhibit the activity of transmembrane protease, serine 2 (TMPRSS2). The TMPRSS2 manipulates the spike proteins of SARS-CoV and MERS-CoV and thus, facilitating viral entry into the cell. By inhibition of TMPRSS2, camostat mesylate blocks the entry of virus in the cell, thereby inhibiting viral replication and infection.[13] It has been reported that the 
SARS-CoV-2 like other coronaviruses enters and infects the host cells through ACE-2 receptor proteins. The binding of the spike proteins of virus and ACE- 2 receptor proteins requires TMPRSS2 and camostat mesylate might be beneficial in the treatment of COVID-19, through the same mechanism.[37] When given to mice following SARS-CoV infection, it reduced the mortality from $100 \%$ to $30-35 \%$. This drug is under the experimental stage for COVID-19 in humans and the outcomes still awaited.

\subsection{Favipiravir}

Another repurposed antiviral drug which is under trial to alleviate COVID-19 is Favipiravir (T-705). It was used against resistant influenza A and B viral infections in Japan and has also been investigated for the treatment of viral haemorrhagic fever (Ebola) and Lassa virus. This compound is a purine nucleoside analogue or a derivative of pyrazine carboxamide. Favipiravir has been approved for clinical trial against SARS-CoV-2 in China, Japan, Italy, and Russia as of June 11, 2020. It acts by inhibiting an enzyme known as RNA-dependent RNA polymerase, responsible for transcription and replication of the viral genome.[28] Phase 1 and 2 of the drug trials have been completed with significant outcomes in terms of efficacy of the drug in COVID-19 patients and reduced time of viral abolition. It is in the third phase of the trial now and the results have yet to be authenticated. [38]

\subsection{Tocilizumab}

Various clinical trials are in progress to test the efficacy of drugs that block a variety of cytokines which are the predecessors of the cytokine storm, a hyperinflammatory response that occurs in the severe cases of COVID-19 resulting in ARDs and MOD. These drugs which belong to the immunomodulatory group are selective cytokine inhibitors which either block the cytokine receptor or inhibit the cytokine itself. One such drug is Tocilizumab used to treat rheumatoid arthritis. Tocilizumab is a recombinant, monoclonal antibody antagonist of the IL-6 receptor. [28] Researchers have found a significant reduction in the number of deaths and the need of mechanical ventilation in patients suffering from severe Pneumonia due to SARS-CoV-2, who received Tocilizumab, either intravenously or subcutaneously along with the standard of care, compared to those who received only the standard of care. $[39,40]$

\subsection{Convalescent plasma therapy}

This is passive-adaptive immunotherapy, that directly targets the pathogen and neutralizes them by immunomodulation. [24] Plasma with hyperimmune antibodies obtained from the recovered patients have been proposed for treating critically ill COVID-19 patients but the effectiveness and safety of convalescent plasma therapy to manage COVID-19 are constrained and uncertain. [41] Recently the results of an open-label, phase II, multicentre, randomized controlled trial (PLACID TRIAL) happening in India were released and according to the reports there was no reduction in the disease progression in terms of severity as well as mortality rate among COVID-19 hospitalized patients. Although some beneficial effects were noted regarding breathing difficulty, need of supportive oxygen therapy, and improvement of general condition, the overall effectiveness of convalescent plasma therapy remains limited against SARS-CoV-2. [42]

\subsection{Herd immunity}

For highly contagious diseases like SARS-CoV-2 infection, it is really hard to achieve herd immunity as a great proportion of the population needs to be immunized either by vaccination or by carrying infection which develops immunity for any future exposure to the specific agent. An approved vaccine for COVID-19 is not available yet and recurrence of COVID-19 infection have been reported in sporadic cases which proves that antibodies develop after the first exposure to SARS-CoV-2 virus might 
not be effective against further exposure to the virus. Many projects for the development of vaccines against SARS-CoV-2 are in progress. Some of the projects which are in phase II and III stage with promising results are as follows:

5.11.1 Moderna. This vaccine trial is being conducted in Seattle, Washington. A messenger RNA (mRNA-1273) vaccine approved from the FDA has been tested through three phases clinical trial. [43] Recently the results are announced by the company that mRNA-1273 is highly effective in generating immunity for COVID-19.

5.11.2. The University of Oxford in England. This vaccine (ChAdOx $1 \mathrm{nCoV}-19)$ trial is based on the use of a modified virus that stimulates the immune system of the host. The clinical trial initiated in April as a joint venture of Oxford University and a biopharmaceutical company AstraZeneca, with more than 500 participants. [43] It is in the third phase of the clinical trial and the results are awaited. Preliminary results of the first two phases revealed that the efficacy and safety of the vaccine. The vaccine generated a strong immune response by increased production of antibodies and enhanced responses from T-cells.

\subsection{Infection Control \& Public Health Measures to Limit Transmission of SARS-CoV-2}

Prevention measures are strongly implemented in the affected countries to reduce transmission in the community. Self-isolation by people and complete lockdown enforced by government authorities in China have played a major role in breaking the chain of COVID-19. [44] Many countries employed the same strategy to stop the spread of this highly contagious virus. There are still aggressive isolation and social distancing measures at some places such as the U.S, India, and some countries of Europe, where the virus keeps on resurging. According to WHO guidelines quarantine of diagnosed COVID-19 patients, isolation of any suspected cases till screening for SARS-CoV-2 comes negative, frequent hand washing, application of sanitizers, use of cloth face coverings and gloves are highly effective precautionary measures against the spread of COVID-19. Elderly and patients with a medical history of chronic non-communicable diseases such as cardiovascular diseases, diabetes, renal failure, cancer, autoimmune diseases, are more vulnerable to contract viral infections with grave prognosis and therefore clear guidelines have been provided by the health authorities to healthcare providers. [45] Healthcare agencies have recommended telemedicine where possible to avoid overcrowding in the hospitals. Strict preventive and self-protective measures should be practised by healthcare staff using N95 respirators, surgical masks, eye protection, face shields, gloves, and isolation gowns to halt the transmission of the virus. [46]

\section{Conclusion}

Some time-tested measures to control the outbreak and spread are to encourage proper hygiene, preventing contact with suspected animal hosts of such viruses and isolation of individuals with a confirmed positive diagnosis while waiting for the development of a specific vaccine and a therapeutic substance to control SARS-CoV-2. More research needs to be done on the timely detection of these diseases and countries need to put protocols in place to restrict the movement of individuals immediately to stop another local outbreak from turning into a global pandemic next time.

\section{Authors' contribution}

Authors UAJ and IR conceptualized and design the study. Author UAJ and IR performed the literature search and filter the relevant research studies and clinical trials. Author UAJ analysed the literature and wrote the original draft. Author IR and SAJ review, edit, and format the draft and all the authors have approved the final manuscript. 


\section{Declaration of Interest}

The authors declare that there is no conflict of interest.

\section{References}

[1] Xie P, Ma W, Tang H, Liu D. Severe COVID-19: A Review of Recent Progress With a Look Toward the Future. Front Public Heal. 2020 May 13;8(189).

[2] Sohrabi C, Alsafi Z, O'neill N, Khan M, Kerwan A, Al-Jabir A, et al. World Health Organization declares global emergency: A review of the 2019 novel coronavirus (COVID19). Int J Surg [Internet]. 2020;76:71-6. Available from: https://doi.org/10.1016/j.ijsu.2020.02.034

[3] Zhang Y-Z, Holmes1 EC. A Genomic Perspective on The Origin and Emergence of SARSCoV2. Cell. 2020;181(2):223-227.

[4] Li H, Liu SM, Yu XH, Tang SL, Tang CK. Coronavirus disease 2019 (COVID-19): current status and future perspectives. Int J Antimicrob Agents. 2020;55(5):105951.

[5] WHO. WHO Coronavirus Disease (COVID-19) Dashboard [Internet]. 2020 [cited 2020 Jun 8]. Available

from: https://covid19. who.int/?gclid=Cj0KCQjwoPL2BRDxARIsAEMm9y9ApKkYIb3ecnPB1zfo uil0dlS9cp1tst9Q3JULEOT7hUv39EUHyYEaA139EALw_wcB

[6] Lu H. Drug treatment options for the 2019-new coronavirus (2019-nCoV). Biosci Trends. 2020;14(1):69-71.

[7] WHO. Solidarity clinical trial for COVID-19 treatments [Internet]. 2020 [cited 2020 Jun 7]. Available from: https://www.who.int/emergencies/diseases/novel-coronavirus-2019/globalresearch-on-novel-coronavirus-2019-ncov/solidarity-clinical-trial-for-covid-19-treatments

[8] Wu A, Peng Y, Huang B, Ding X, Wang X, Niu P, et al. Genome Composition and Divergence of the Novel Coronavirus (2019-nCoV) Originating in China. Cell Host Microbe. $2020 \mathrm{Mar}$ $11 ; 27(3): 325-8$.

[9] Petrosillo N, Viceconte G, Ergonul O, Ippolito G, Petersen E. COVID-19, SARS and MERS: are they closely related? Clin Microbiol Infect. 2020 Jun 1;26(6):729-34.

[10] Li Q, Guan X, Wu P, Wang X, Zhou L, Tong Y, et al. Early Transmission Dynamics in Wuhan, China, of Novel Coronavirus-Infected Pneumonia. N Engl J Med. 2020 Jan 29;382:1199-207.

[11] Galbadage T, Peterson BM, Gunasekera RS. Does COVID-19 Spread Through Droplets Alone? Front Public Heal [Internet]. 2020 Apr 24 [cited 2020 Jun 4];8(163). Available from: https://www.frontiersin.org/article/10.3389/fpubh.2020.00163/full

[12] Guo YR, Cao QD, Hong ZS, Tan YY, Chen SD, Jin HJ, et al. The origin, transmission and clinical therapies on coronavirus disease 2019 (COVID-19) outbreak- A n update on the status. Mil Med Res. 2020 Mar 13;7(11).

[13] Hoffmann M, Kleine-Weber H, Schroeder S, Krüger N, Herrler T, Erichsen S, et al. SARS-CoV2 Cell Entry Depends on ACE2 and TMPRSS2 and Is Blocked by a Clinically Proven Protease Inhibitor. Cell. 2020;181(2):271-80.

[14] Li X, Geng M, Peng Y, Meng L, Lu S. Molecular immune pathogenesis and diagnosis of COVID19. J Pharm Anal. 2020 Apr 1;10(2):102-8.

[15] Nile SH, Nile A, Qiu J, Li L, Jia X, Kai G. COVID-19: Pathogenesis, cytokine storm and therapeutic potential of interferons. Cytokine Growth Factor Rev. 2020 Jun 1;53:66-70.

[16] Patel SK, Saikumar G, Rana J, Dhama J, Yatoo MI, Tiwari R, et al. Dexamethasone: A boon for critically ill COVID-19 patients? Travel Med Infect Dis [Internet]. 2020 Sep 1 [cited 2020 Sep 15];37:101844. Available from: https:/www.ncbi.nlm.nih.gov/pmc/articles/PMC7416109/

[17] Wang Y, Wang Y, Chen Y, Qin Q. Unique epidemiological and clinical features of the emerging 2019 novel coronavirus pneumonia (COVID-19) implicate special control measures. J Med 
Virol [Internet]. 2020 Mar 5 [cited 2020 Mar 22];92:568-576. Available from: http://www.ncbi.nlm.nih.gov/pubmed/32134116

[18] Siordia JA. Epidemiology and clinical features of COVID-19: A review of current literature. J Clin Virol. 2020 Jun 1;127.

[19] Chang D, Lin M, Wei L, Xie L, Zhu G, Dela Cruz CS, et al. Epidemiologic and Clinical Characteristics of Novel Coronavirus Infections Involving 13 Patients Outside Wuhan, China. JAMA - J Am Med Assoc. 2020 Mar 17;323(11):1092-3.

[20] Huang C, Wang Y, Li X, Ren L, Zhao J, Hu Y, et al. Clinical features of patients infected with 2019 novel coronavirus in Wuhan, China. Lancet. 2020 Feb 15;395(10223):497-506.

[21] Zarei A, Fardood T, Moradnia |farzaneh, Ramazani ali. A Review on coronavirus family persistency and considerations of novel type, covid-19 features. Eurasian Chem Commun [Internet]. 2020 [cited 2020 Jun 5];2:798-811. Available from: http:/echemcom.com

[22] Singhal T. A Review of Coronavirus Disease-2019 (COVID-19). Indian J Pediatr. 2020 Mar $13 ; 87(4): 281-6$.

[23] Baden LR, Rubin EJ. Covid-19 - The search for effective therapy. N Engl J Med [Internet]. 2020 May 7 [cited 2020 Jun 2];382(19):1851-2. Available from: http://www.nejm.org/doi/10.1056/NEJMe2005477

[24] Lotfi M, Hamblin MR, Rezaei N. COVID-19: Transmission, Prevention, and Potential Therapeutic Opportunities. Clin Chim Acta [Internet]. 2020 May 28 [cited 2020 Jun 5];508:254-66. Available from: http://www.ncbi.nlm.nih.gov/pubmed/32474009

[25] Yang Z, Liu J, Zhou Y, Zhao X, Zhao Q, Liu J. The effect of corticosteroid treatment on patients with coronavirus infection: a systematic review and meta-analysis. J Infect [Internet]. 2020 [cited 2020 Jun 5];81(1):E13-E20,. Available from: https://doi.org/10.1016/j.jinf.2020.03.062

[26] Ledford H. Coronavirus breakthrough: dexamethasone is first drug shown to save lives. Vol. 582, Nature. NLM (Medline); 2020. p. 469.

[27] Mahase E. Covid-19: Demand for dexamethasone surges as RECOVERY trial publishes preprint. BMJ. 2020 Jun 23;369:m2512.

[28] Delang L, Neyts J. Medical treatment options for COVID-19. Eur Hear JournalAcute Cardiovasc Care [Internet]. 2020 May 4 [cited 2020 Aug 28];9(3):219-214. Available from: /pmc/articles/PMC7235633/?report=abstract

[29] Beigel JH, Tomashek KM, Dodd LE, Mehta AK, Zingman BS, Kalil AC, et al. Remdesivir for the Treatment of Covid-19 - Preliminary Report. N Engl J Med [Internet]. 2020 May 22 [cited 2020 Aug 29]; Available from: https://www.nejm.org/doi/full/10.1056/NEJMoa2007764

[30] Bimonte S, Crispo A, Amore A, Celentano E, Cuomo A, Cascella M. Potential antiviral drugs for SARS-Cov-2 treatment: Preclinical findings and ongoing clinical research [Internet]. Vol. 34, In Vivo. International Institute of Anticancer Research; 2020 [cited 2020 Aug 28]. p. 1597602. Available from: http://iv.iiarjournals.org/content/34/3_supp1/1597.full

[31] Liu X, Wang XJ. Potential inhibitors against 2019-nCoV coronavirus M protease from clinically approved medicines. J Genet Genomics [Internet]. 2020 Feb 20 [cited 2020 Aug 31];47(2):119-21. Available from: https://www.ncbi.nlm.nih.gov/pmc/articles/PMC7128649/

[32] Nukoolkarn V, Lee VS, Malaisree M, Aruksakulwong O, Hannongbua S. Molecular dynamic simulations analysis of ritronavir and lopinavir as SARS-CoV 3CLpro inhibitors. J Theor Biol. 2008 Oct 21;254(4):861-7.

[33] Li L, Li R, Wu Z, Yang X, Zhao M, Liu J, et al. Therapeutic strategies for critically ill patients with COVID-19. Ann Intensive Care [Internet]. 2020 Dec 1 [cited 2020 Jun 5];10(45). Available from: https://annalsofintensivecare.springeropen.com/articles/10.1186/s13613020-00661-z

[34] Juurlink DN. Safety considerations with chloroquine, hydroxychloroquine and azithromycin in the management of SARS-CoV-2 infection. Vol. 192, CMAJ. Canadian Medical Association; 2020. p. E450-3. 
[35] Lee JS, Shin EC. The type I interferon response in COVID-19: implications for treatment [Internet]. Nature Reviews Immunology. Nature Research; 2020 [cited 2020 Sep 5]. p. 1-2. Available from: https://doi.org/10.1038/

[36] Davoudi-Monfared E, Rahmani H, Khalili H, Hajiabdolbaghi M, Salehi M, Abbasian L, et al. Efficacy and safety of interferon $\beta$-1a in treatment of severe COVID-19: A randomized clinical trial. Antimicrob Agents Chemother [Internet]. 2020 May 30 [cited 2020 Sep 5];2020.05.28.20116467. Available from: https://doi.org/10.1101/2020.05.28.20116467

[37] The Impact of Camostat Mesilate on COVID-19 Infection - Full Text View - ClinicalTrials.gov [Internet]. [cited $2020 \quad$ Sep 5]. Available from: https://clinicaltrials.gov/ct2/show/NCT04321096

[38] Favipiravir in Hospitalized COVID-19 Patients - Full Text View - ClinicalTrials.gov [Internet]. [cited 2020 Sep 1]. Available from: https://clinicaltrials.gov/ct2/show/NCT04359615

[39] Toniati P, Piva S, Cattalini M, Garrafa E, Regola F, Castelli F, et al. Tocilizumab for the treatment of severe COVID-19 pneumonia with hyperinflammatory syndrome and acute respiratory failure: A single center study of 100 patients in Brescia, Italy. Autoimmun Rev. $2020 \mathrm{Jul}$ $1 ; 19(7): 102568$.

[40] Guaraldi G, Meschiari M, Cozzi-Lepri A, Milic J, Tonelli R, Menozzi M, et al. Tocilizumab in patients with severe COVID-19: a retrospective cohort study. Lancet Rheumatol [Internet]. 2020 Aug 1 [cited 2020 Aug 30];2(8):e474-84. Available from: www.thelancet.com/rheumatology

[41] Devasenapathy N, Ye Z, Loeb M, Fang F, Najafabadi BT, Xiao Y, et al. Efficacy and safety of convalescent plasma for severe COVID-19 based on evidence in other severe respiratory viral infections: a systematic review and meta-analysis. Can Med Assoc J [Internet]. 2020 May 22 [cited 2020 Jun 5];192(27):E745-55. Available from: http://www.ncbi.nlm.nih.gov/pubmed/32444482

[42] Agarwal A, Mukherjee A, Kumar G, Chatterjee P, Bhatnagar T. Convalescent plasma in the management of moderate COVID-19 in India: An open-label parallel-arm phase II multicentre randomized controlled trial (PLACID Trial). medRxiv [Internet]. 2020 [cited 2020 Sep 20];2020.09.03.20187252. Available from: https://doi.org/10.1101/2020.09.03.20187252

[43] Slaoui M, Hepburn M. Developing Safe and Effective Covid Vaccines - Operation Warp Speed's Strategy and Approach. N Engl J Med [Internet]. 2020 Aug 26 [cited 2020 Aug 29];NEJMp2027405. Available from: http://www.nejm.org/doi/10.1056/NEJMp2027405

[44] Cyranoski D. What China's coronavirus response can teach the rest of the world. Nature [Internet]. 2020 [cited 2020 Mar 26];579(7800):479-80. Available from: https://www.nature.com/articles/d41586-020-00741-x NS -

[45] Cherian R, Poh KK. At the "heart" of the COVID-19 outbreak: early cardiac implications and mitigating strategies ONLINE FIRST PUBLICATION. SMJ Singapore Med J [Internet]. 2020 [cited 2020 Apr 22];61(7):373-4. Available from: http://www.smj.org.sg/online-first

[46] Del Rio C, Malani PN. COVID-19 - New Insights on a Rapidly Changing Epidemic. J Am Med Assoc. 2020 Apr 14;323(14):1339-40. 